\title{
marges Marges
}

revue d'art contemporain Revue d'art contemporain

$10 \mid 2010$

Déplacements des pratiques artistiques

\section{Théâtralité et arts visuels : le paradoxe du} spectateur. Autour « The World as a Stage » et "Un teatre sense teatre "

Theatricality and Visual Arts: The Paradox of the Viewer

\section{Laure Fernandez}

\section{(2) OpenEdition \\ Journals}

Édition électronique

URL : http://journals.openedition.org/marges/490

DOI : $10.4000 /$ marges. 490

ISSN : 2416-8742

Éditeur

Presses universitaires de Vincennes

\section{Édition imprimée}

Date de publication : 15 avril 2010

Pagination : 25-36

ISBN : 978-2-84292-254-2

ISSN : $1767-7114$

Référence électronique

Laure Fernandez, "Théâtralité et arts visuels : le paradoxe du spectateur. Autour " The World as a Stage » et « Un teatre sense teatre » », Marges [En ligne], 10 | 2010, mis en ligne le 15 avril 2010, consulté le 19 avril 2019. URL : http://journals.openedition.org/marges/490 ; DOI : 10.4000/ marges.490 


\section{Théâtralité et arts visuels: le paradoxe du spectateur. Autour «The World as a Stage» et «Un teatre sense teatre»}

La notion de théâtralité [theatricality], apparue avec la modernité puis théorisée dans les années 1960, connaît un regain d'intérêt dans nombre de disciplines artistiques. Si la notion est apparue chez les praticiens et théoriciens du théâtre, c'est depuis quelques années dans le champ des arts visuels que sa vitalité est la plus remarquable. Ainsi, le vocabulaire et la métaphore peuvent servir d'outils, voire de modèle théorique et esthétique, que la théâtralité soit utilisée comme une référence positive ou au contraire dans une logique que l'on pourrait qualifier de «théâtrophobique». Témoignent de ce phénomène de migration du théâtral vers les arts visuels des exemples divers, dont le plus connu reste certainement l'usage que fait de cette notion Michael Fried dès la fin des années 1960 aux États-Unis, alors même que s'y formulent de nouveaux questionnements sur l'autonomie des arts. Cette construction d'un discours historique et critique autour de la notion de théâtralité semble intimement liée à la réflexion, aujourd'hui très active, sur les déplacements entre les arts et la place du spectateur. Du côté des œuvres, nous sommes face à un double constat: d'une part, la théâtralité est utilisée comme cadre («mettre en scène»: installer dans le temps et l'espace), de l'autre, elle est inversement utilisée comme un processus de dissolution du cadre (théâtraliser pour «intégrer» à tout prix le spectateur). Des 
/ 1 « Un teatre sense teatre » a été présenté au MACBA de Barcelone du 25 mai au 11 septembre 2007 et "The World As a Stage» à la Tate Modern de Londres du 24 octobre 2007 au $1^{\mathrm{er}}$ janvier 2008.

/ 2 Le mot s'étant banalisé, il est important de distinguer ce qui relève historiquement de l'usage effectif de la notion de théâtralité des conceptions du théâtre formulées autrefois par des auteurs sans recourir au mot, que certains lecteurs contemporains rebaptisent postérieurement "théâtralité». expositions récentes telles que "Un teatre sense teatre » ou encore "The World as a Stage/1 » ont ainsi montré combien les artistes du $20^{\mathrm{e}}$ et $21^{\mathrm{e}}$ siècle se sont emparés du théâtral - cet emprunt pouvant être thématique, mais aussi, de façon plus intéressante, structurel - pour parfois mieux s'en écarter et repenser ainsi leur propre pratique. Nous nous intéresserons à quelques exemples précis de ce déplacement d'un double point de vue, théorique et esthétique. Dans un premier mouvement, il s'agira de redéfinir le terme de théâtralité, en étudiant son évolution au sein du théâtre bien sûr, mais aussi dans les arts visuels. Cette approche devrait permettre de mieux saisir et analyser la complexité contemporaine de la notion et d'étudier un phénomène particulier de déplacement. Dans un second mouvement, il s'agira de s'intéresser à quelques propositions artistiques «non théâtrales» au sens strict, que la notion de théâtralité permet d'éclairer. Les deux expositions précédemment citées nous serviront d'objet d'étude dans ce cadre.

\section{La théâtralité: entre quête et phobie}

C'est assez tardivement que la notion de théâtralité apparaît dans les discours théoriques sur le théâtre. En effet, si l'idée n'est pas nouvelle et peut transparaître déjà d'une certaine manière chez Aristote lorsqu'il tente de définir l'essence du théâtre, la théâtralité est avant tout une préoccupation moderne/2. L'étude de différents dictionnaires d'usage français a montré que l'apparition de la notion semble difficile à dater précisément. Ainsi Le Robert qui situait, il y a encore quelques années, son émergence dans les années 1950, date désormais sa première acception en 1842, tout comme le Trésor de la Langue française. Il apparaît également que la notion ne se trouve dans les dictionnaires, jusque dans les années 1980, qu'en sous-catégorie, comme dérivé de l'adjectif théâtral, voire pas du tout. Dès 1971 cependant, Pierre Gilbert lui consacre un bref article dans son Dictionnaire des mots nouveaux, lui donnant la définition plurielle et vague de «qualités théâtrales d'une œuvre». Ce qui ressort de l'ensemble des définitions, c'est que la théâtralité n'y est envisagée que par rapport à un modèle théâtral et qu'elle se rapporterait exclusivement à la scène: les articles parlent de «moyens spécifiquement scéniques », font référence à la lumière, au décor, à la gestuelle, au ton, aux éléments stylistiques et structurels de l'art théâtral, à sa représentation, et non pas à sa «forme littéraire ». Il est intéressant de constater que seul le Trésor de la Langue française rattache la notion de théâtralité exclusivement au théâtre, quand les autres articles font référence à d'autres pratiques artistiques (on 
parle par exemple de la «théâtralité d'un opéra», de la «conformité d'une œuvre plastique ou musicale» aux règles du théâtre, etc.). Par ailleurs, l'ensemble de ces définitions témoigne d'une approche du théâtre directement héritée des courants sémiologiques et sémiotiques: la théâtralité est envisagée comme plurielle, composée d'un ensemble d'éléments qui, pris seuls ou à plusieurs, signeraient la dimension théâtrale d'une œuvre.

Dans le champ propre au théâtre, l'idée de théâtralité apparaît au moment où le cinéma vient bouleverser les spécificités du théâtre et où ce dernier tente de se dégager de la littérature, c'est-à-dire à un moment où le théâtre cherche à se définir tout d'abord en tant qu'Art, puis en tant que forme autonome. Dès ses origines, la notion prend une double signification, à la fois positive et négative - proche de ce qui sera désigné plus tard comme le théâtralisme. En 1908, le Russe Nicolas Evreinoff est l'un des premiers praticiens à consacrer une réflexion spécifique à la notion dans son essai Apologie de la théâtralité. II affirme l'aspect conventionnel du théâtre au moment où le naturalisme règne en maître et propose un élargissement précurseur du concept à la lumière de la sociologie et de la psychologie. La théâtralité serait un instinct pré-esthétique apparu bien avant le théâtre, dont «le plus haut degré» correspondrait «dans l'homme et la société, au plus haut degré de civilisation/3». La théâtralité, pour Evreinoff, serait intrinsèque à l'être vivant et se manifesterait tant dans la vie qu'au théâtre. Au centre des problématiques des avant-gardes modernes, la théâtralité devient intimement liée à une réflexion sur la représentation, et plus exactement sur la mise en scène, recouvrant une valeur quasi symbolique: «La modernité conçoit la théâtralité comme manque, désir et recherche de théâtre, au lieu de faire du théâtre un art défini et accompli /4. ».

Dès lors, la théâtralité devient l'objet d'une quête: «rethéâtraliser» le théâtre (l'apogée en étant, pour beaucoup, le théâtre russe des années 1920-1930, et plus particulièrement celui de Meyerhold qui développera l'idée de lois théâtrales uniques). C'est que les utopies d'un Wagner ont laissé leurs marques: si le théâtre est le lieu privilégié de rassemblement (voire de fusion) d'arts comme la musique, la danse, la littérature, que lui reste-t-il en propre? Qu'est-ce qui permet alors d'estimer qu'une œuvre a des qualités théâtrales? Tout au long de la première moitié du $20^{\mathrm{e}}$ siècle, différents praticiens tenteront d'ériger le théâtre comme un art indépendant, ce que la mise en scène semble, dans un premier temps, leur offrir. L'idée de théâtralité permet ainsi à un certain nombre d'hommes de théâtre de contrer leur propre méfiance anti-théâtrale ou, tout du moins, leur mécontentement vis-à-vis des formes alors actuelles du thêâtre.
/3 Introduction à l'ouvrage de Nicolas Evreinoff, Le Théâtre dans la vie ( $2^{\mathrm{e}}$ éd.), Paris, Stock, 1930, p. XIV.

/4 Geneviève Jolly et Muriel Plana, "Théâtralité », dans Jean-Pierre Sarrazac (sld.), Lexique du drame moderne et contemporain, Belval, Circé, coll. Poche, 2005 , p. 215. 
/5 Roland Barthes, "Le théâtre de Baudelaire», Essais critiques, dans Euvres complètes II. Livres, textes, entretiens. 1962-1967, Paris, Seuil, 2002, p. 304. Notons que ce n'est pas l'éviction du texte que clament respectivement ces auteurs, mais sa mise à hauteur des autres signes qui forment le tout théâtral: un théâtre libéré de la littérature.

/6 Michel Bernard, L'Expressivité du corps. Recherche sur les fondements de la théâtralité, Paris, Jean-Pierre Delarge, coll. Corps et culture, 1976. Michèle Fèbvre (Danse contemporaine et théâtralité, Paris, Chiron, 1995), dans cette lignée, avancera l'idée d'une théâtralité transcendantale, c'est-à-dire détachée du théâtre, située en amont des pratiques afin de sortir de la tautologie théâtralité égale théâtre.

/ 7 Josette Féral, "La Théâtralité. Recherche sur la spécificité du langage théâtral », Poétique, septembre 1988 , respectivement p. 348 , p. 349 et p. 350

/8 ibid., p. 358.

/9 Bernard Dort "La Représentation émancipée », dans La Représentation émancipée, Arles, Actes Sud, coll. Le Temps du théâtre, 1988, p. 178 et p. 183.
Cela leur permet de réaffirmer ainsi - par ce qui est plus qu'un léger décalage notionnel - leurs quêtes et leurs croyances. Les exemples en sont nombreux, de Brecht à Jarry et son fameux texte "De l'inutilité du théâtre au théâtre », jusqu'à Artaud déplorant la disparition de la scène dans les démarches occidentales. La première approche spécifiquement théorique sera sémiologique: pour Roland Barthes, en 1964, la théâtralité doit être envisagée par soustraction («[la théâtralité,] c'est le théâtre moins le texte/5»), puis par division (différents signes spécifiquement théâtraux). En 1976, alors que le règne de la mise en scène est plus que jamais remis en cause, Michel Bernard propose une approche épistémologique et pense la théâtralité comme une propriété du corps en jeu: des éléments scéniques, la théâtralité se déplace vers l'acteur (le performeur/6). Les années 1980 achèvent de détacher la théâtralité de la mise en scène théâtrale, prenant pour la première fois explicitement en compte le spectateur dans un contexte de crise du théâtre où les créations sont de plus en plus interdisciplinaires. Ainsi, pour Josette Féral, on peut envisager la théâtralité comme une "propriété du quotidien", un travail prioritairement spatial qui surgit du «savoir du spectateur dès lors qu'il a été informé de l'intention de théâtre à son adresse »: «Plus qu'une propriété dont il serait possible d'analyser les caractéristiques, [la théâtralité] semble être un processus, une production qui tient tout d'abord au regard, regard qui postule et crée un espace autre qui devient espace de l'autre - espace virtuel, cela va de soi et laisse place à l'altérité des sujets et à l'émergence de la fiction. Cet espace est le résultat d'un acte conscient qui peut partir soit du performeur lui-même [...] soit du spectateur dont le regard crée un clivage spatial où peut émerger l'illusion [...] et qui peut porter sans distinction sur les évènements, les comportements, les corps, les objets et l'espace autant du quotidien que de la fiction /7. ».

La condition sine qua non de la théâtralité, selon Josette Féral, serait donc la présence d'un cadre de l'action, spatial - mais surtout et avant tout structurel - basé sur un contrat tacite, celui d' "assister à un acte de re-présentation inscrit dans une temporalité autre que celle du quotidien, où le temps est comme suspendu et pour ainsi dire réversible, qui impose à l'acteur le retour toujours possible à sa position de départ/8 ». Bernard Dort, la même année, reviendra sur le texte de Barthes en faisant de la théâtralité dans ce contexte une "polyphonie signifiante ouverte sur le spectateur»: "La théâtralité, alors, n'est plus seulement cette "épaisseur de signes" dont parlait Roland Barthes. Elle est aussi le déplacement de ces signes, leur impossible conjonction, leur confrontation sous le regard du spectateur de cette représentation émancipée/9. ». 
Parallèlement à cette quête interne du théâtre la modernité artistique développe sa propre définition de la théâtralité sous l'influence des recherches wagnériennes, voyant en elle l'ennemi juré de la volonté de purification des disciplines qui s'instaure alors. Cette théâtrophobie latente atteindra son point culminant dans les critiques adressées à l'art minimal par Michael Fried dans le désormais célèbre «Art and Objecthood» (1967). Car si la modernité artistique se définit par ce «nouveau laocoonisme» greenbergien qu'est la spécificité de chaque discipline par exclusion mutuelle, le théâtre - art «singulierpluriel » s'il en est/10 - ne peut être moderniste - il ne peut être un art et doit être soigneusement tenu à distance des autres pratiques artistiques. De plus, son dispositif spécifique et constitutif de co-présence scène/salle signe la particularité - forcément négative pour la modernité - de cet art dont la forme définitive ne se déploie que dans cette réunion. L'exigence de spécificité et la motivation autoréférentielle modernistes seront dès lors mises à mal par les «objets spécifiques» de l'art minimal, dont le principal enjeu est justement l'indétermination et l'incomplétude. Conséquence immédiate: ce courant qui apparaît alors sur le devant de la scène artistique américaine - parce qu'il pense dans sa conception même la relation regardant/regardé en prenant en compte les paramètres spatiaux et temporels de présentation de l'œuvre - est rapidement catalogué par Fried comme le "symptôme d'une sensibilité corrompue et pervertie par le théâtre/11 ». C'est à partir de cette donnée fondamentale que Fried va développer sa critique de la théâtralité dans ce qu’il nomme l'art littéraliste à partir de trois idées:

1) «Le succès, et même la survie, des formes artistiques dépend de plus en plus de leur capacité à mettre le théâtre en échec.»

2) «L'art dégénère à mesure qu'il approche une condition qui est celle du théâtre.»

3) «Les concepts de qualité et de valeur [...] prennent un sens, ou prennent tout leur sens, à l'intérieur, exclusivement, des arts individuels. Ce qui existe entre les arts relève du théâtre /12. ».

Au lieu de n'être qu'une querelle passagère (car le texte de Fried n'était, après tout, qu'une critique), cette déclaration fait date, parce qu'elle n'est pas une lubie soudaine et isolée mais le résultat d'une importante lignée critique antithéâtrale (n'oublions pas que Fried s'inspire des théories de Diderot) dont les retombées se font encore ressentir aujourd'hui/13. Cette querelle témoigne finalement, en négatif, de la force de ce déplacement du modèle théâtral vers d'autres théories; déplacement qui a permis de penser tout un changement quasiment paradigmatique de la conception de l'œuvre d'art autour du spectateur. Si la peinture américaine des années 1950
/10 Marie-Christine Lesage (relisant Jean-Luc Nancy), «L'Interartistique: une dynamique de la complexité », $R$ evue d'Études Théâtrales, $n^{\circ} 13$, printemps 2008 , p. 11-26.

/11 Jacques Sato, "Littéralité et théâtralité » dans Louis Dieuzayde (sld), Le Langage s'entend mais la pensée se voit, Aix-en-Provence, PUP, coll. Théorie et pratique des arts, 2007, p. 164.

/12 Michael Fried, "Art et Objectité » (1967), Contre la théâtralité. Du minimalisme à la photographie contemporaine, Paris, Gallimard, coll. NRF Essais, 2007, respectivement p. $133-134$, p. 135 et p. 136.

/13 Voir aussi Michel Poivert sur les relations photographie/théâtralité. Selon lui, tout un pan de l'histoire de la photographie ne s'est pas écrit, conséquence de cette théâtrophobie de près d'un siècle. 
/14 Quelqu'un comme Féral construira sa définition en relisant Fried, c'est-à-dire en pensant la théâtralité des arts scéniques à la lumière de l'approche proposée par les arts visuels.

15 Rappelons que la thêâtralité n'est pas le théâtre; en conséquence, ce qui se joue ici n'est pas la question de l'interdisciplinarité théâtre/arts plastiques.

/16 Cette classification autour de différentes notions est un outil de travail, les œuvres n'étant évidemment pas aussi univoques.

/17 Josette Féral, "La Théâtralité.

Recherche sur la spécificité du langage théâtral », op. cit., p. 356. semblait illustrer un certain triomphe de la pensée moderniste, le bouleversement réel qu'ont provoqué dix ans plus tard les premières œuvres minimalistes crée l'événement et se fait ressentir aujourd'hui encore : l'expérience prime, avec une prise en compte toute nouvelle du corps du spectateur dans la relation esthétique. Ce que certains perçoivent comme une véritable victoire de la phénoménologie sur l'œil désincarné. Alors qu'elle n'apparaît explicitement que très tardivement dans les théories théâtrales, la figure du spectateur fonde dès la fin des années 1960, chez Fried, une conception de la théâtralité/14. Ainsi, malgré (ou grâce à) la doctrine moderniste, nombre de propositions artistiques intègreront dès la seconde moitié du $20^{\mathrm{e}}$ siècle la complexité de la théâtralité dans des pratiques diverses, souvent en marge des dispositifs traditionnels (installations, performance, happening, event, art in situ...) : il existerait une place, et non des moindres, pour le théâtral dans l'art et dans les musées. C'est à cet endroit que se situent les expositions auxquelles nous nous intéresserons.

\section{Quand le contemporain réhabilite le théâtral: la scène en question}

Contenant intrinsèquement, dans ses différents usages, un déplacement du théâtre par rapport à lui-même /15, la théâtralité permettrait de penser d'une part les glissements entre les pratiques artistiques, d'autre part les glissements - structurels avant tout - au sein même d'une pratique artistique particulière à la lumière du théâtral. II semble intéressant, pour mieux observer ce phénomène, de revenir sur quelques notions-clés afin de repérer un premier niveau de manifestation de la théâtralité dans des œuvres contemporaines: une référence au théâtre/théâtral dans le contenu et la forme, qu'on désignera comme cognitif/16.

La première notion serait la plus évidente pour le sens commun, à savoir la dichotomie fictif/réel qui sous-tend implicitement l'idée de théâtre. Dans l'histoire du théâtre, on le sait, le terme théâtral a oscillé entre une définition péjorative, synonyme de faux dont l'acteur doit se méfier (c'est le cas chez Stanislavski par exemple) et une définition synonyme de pratique autonome, nécessaire pour "affirmer le "théâtral" comme distinct de la vie et distinct du réel», " comme la condition sine qua non de la théâtralité sur la scène /17 " (chez Meyerhold notamment). Cet aspect proprement dialectique de la scène, impliquant pour le spectateur une dialectique du regard, semble être une manifestation fondamentale de la théâtralité dans les arts visuels. Que cet aspect serve l'interrogation des notions 
de représentation - avec parfois comme la reprise de la question ancestrale de la mimesis, mais le plus souvent comme interrogation des modalités de présentation de l'œuvre même -, d'espace - la distinction/non-distinction des frontières regardant/regardé, espace de l'action/espace de l'audience - et surtout de jeu: qui du regardant ou du regardé fait l'événement? Quand et où commence et finit le fictif? Ces questions peuvent évidemment se recouper au sein d'une même œuvre.

L'exposition de la Tate Modern, «The World as a Stage», regroupait ainsi un panel de propositions artistiques de la fin $\mathrm{du} 20^{\mathrm{e}} /$ début $\mathrm{du} 21^{\mathrm{e}}$ siècle de seize artistes travaillant autour de ces problématiques. J'en présenterai ici quelques exemples. This is New (2003) de l'Anglais Tino Sehgal jetait le visiteur dans le trouble dès son entrée dans l'exposition quand le contrôle des billets se transformait en un échange improbable et absurde: le gardien accueillait chaque visiteur en déclamant à haute voix un gros titre extrait d'un journal de son choix (avec des déclarations portant, selon les jours, sur le réchauffement climatique ou la virginité de Camilla), avec pour mot d'ordre, si le visiteur répondait, de simplement énoncer le titre de l'œuvre, l'année de sa création et le nom de l'artiste, s'il ne répondait pas, d'en rester là (et «l'œuvre» n'est, alors, pas révélée).

Dans un même registre, le visiteur était invité par une bande-annonce à se rendre à une date précise au Borough Market de Londres pour découvrir le nouveau film de l'acteur Jude Law. Film (2000-2007) de l'artiste polonais Pawel Althamer s'avérait être en réalité, pour le visiteur venu au Borough Market, une sorte de happening (realtime movie, dit l'artiste) dans lequel le spectateur se trouvait mêlé à l'action (par ailleurs non-action puisque rien de particulier ne se passait) et prenait conscience d'être au cœur du film simplement en reconnaissant des éléments qu'il avait vus dans la bande-annonce (des figurants, des situations, Jude Law achetant un poisson pour les plus chanceux...). Ainsi, chez Sehgal, la théâtralité n'apparaît que si le visiteur modifie rapidement son regard porté sur la fonction habituelle du gardien. Chez Althamer, elle demande du temps, de la mémoire (reconnaître les figurants aperçus sur la bande-annonce pour comprendre qu'il n'y aura pas de film, au sens traditionnel du terme). Ce qui semble intéresser ici les artistes est cette zone floue qui émerge dès lors que le spectateur est inclus dans l'espace de l'action (elle-même souvent minime et plus proche du non-événement que d'une véritable action), délaissant finalement sa posture même de spectateur.

Dans d'autres cas, c'est l'espace lui-même qui prime et vient cadrer, presque contre son gré, le visiteur dans un espace de jeu. Il en est 
ainsi, par exemple, de Curtain (2007) de l'artiste allemande Ulla Von Brandenburg et de Arena (1997) de l'artiste américaine Rita McBride. Dans la première installation, le visiteur se trouve confronté à un rideau de théâtre se fermant - reconstitution de celui conçu par Walpole Champneys en 1932 pour le Royal Shakespeare Theatre de Stratford-sur-Avon - ne sachant plus s'il se trouve du côté de la scène ou de la salle. Dans la deuxième installation, son statut dans l'exposition se trouve inversé quand, entrant dans une des salles de la Tate, il se retrouve sur une scène, face à d'autres gens assis sur des gradins qui assistent à son entrée. Dans ces exemples, la théâtralité émerge du spectateur dès lors que celui-ci prend conscience de sa participation à une expérience commune/sociale de l'ordre du jeu. Ces démarches aujourd'hui nombreuses, souvent ludiques, quand elles ne sont pas anecdotiques, rejoignent en les détournant, voire en les contredisant (une utilisation presque paradoxale de la thêâtralité comme dissolution du cadre) les approches théâtrales des années 1980. Ces approches cherchaient à détacher la théâtralité du théâtre et de la mise en scène pour en faire une propriété de la scène, au sens structurel du terme, soit de tout événement qui, dans un lieu et un temps donnés, rassemble un regardé et un regardant.

Un deuxième aspect, très présent chez les artistes réunis à la Tate Modern, est lié à une dimension plus politique du théâtre, la théâtralité servant alors à la fois d'outil analytique et de terrain pour des expérimentations artistiques, mais aussi sociales, relisant dans certains cas les théories debordiennes. Reprenant pour titre le motif shakespearien de As You Like It - «All the world's a stage, and all the men and women merely players» [le monde entier est une scène et les hommes et femmes ne sont que des acteurs] - l'exposition «The World as a Stage», outre sa volonté de réhabilitation artistique du théâtral, tend à s'interroger sur ce déplacement social et anthropologique, héritage du theatrum mundi latin: qu'en est-il de la théâtrocratie dans notre société? Quelle place pour l'homme individuel et la communauté? Que peut encore l'art dans une société du spectacle? Témoignent de ces interrogations trois travaux très différents du Polonais Cezary Bodzianowski, de l'Anglais Jeremy Deller et de l'Américaine Catherine Sullivan. Se réclamant à la fois d'une tradition surréaliste et dadaïste et de l'humour trivial des caméras cachées, Bodzianowski propose une action quasi absurde, prenant littéralement au pied de la lettre la thématique même de l'exposition avec sa performance Flying Helmet (2007). En sortant de la Tate Modern, le spectateur découvre l'artiste accroché à une nacelle, mesurant et calculant la surface totale du Théâtre du Globe, ce théâtre shakespearien reconstitué sur les bords de la Tamise, à deux pas du musée 
d'art contemporain, dans le but de comparer ensuite ses mesures avec celles d'un autre globe, la Terre, posant ainsi un regard ironique sur un symbole national anglais en même temps que sur un certain nombrilisme patriotique ambiant. Dans The Battle of Orgreave (2001), Jeremy Deller, vainqueur du prix Turner en 2004, remet en scène et filme, environ vingt ans après, les violents affrontements qui ont opposé mineurs et policiers le 18 juin 1984. Pour ce faire, il invite d'anciens mineurs et policiers impliqués à l'époque dans le conflit à participer, aux côtés d'acteurs, à ce qui devient à la fois une reconstitution (au sens où l'on reconstitue la scène d'un meurtre pour mieux la comprendre et la résoudre) et un acte de réécriture de l'histoire par ceux qui en étaient les véritables acteurs (la BBC ayant par exemple été obligée, à l'époque, de s'excuser publiquement d'une version des faits orientée de manière à présenter les mineurs comme ayant provoqué le début des violences). Une grande partie de The Battle of Orgreave, prise en charge par le réalisateur Mike Figgis, est consacrée à la présentation de la préparation prudente de cette reconstitution, résultat de plus d'un an de recherches à partir d'archives et d'entretiens. Au centre, cette question: que signifie rejouer le passé récent? Comment faire la part des vérités et des fictions dans les événements historiques? La théâtralité, outre un processus de représentation, devient dans un tel cas un «processus consistant à placer, cadrer, situer /18 ». Dans une toute autre démarche, Catherine Sullivan, issue de la performance, explore quant à elle le théâtre-même comme forme visuelle et structurelle servant une perspective à la fois esthétique et anthropologique, en plaçant au cœur de ses œuvres souvent baroques et excessives (films, installations, performances) l'acte même de performeur. Tout ce qui signe la théâtralité dans un des sens premiers du terme (les éléments spécifiquement théâtraux liés ici à l'acteur: gestes, attitudes, costumes, techniques de jeu...) est décortiqué, isolé, réintroduit autrement, répété inlassablement, essoré, pourrait-on dire, en-dehors du cadre du théâtre jusqu'à perdre toute signification ou possibilité d'identification du spectateur et produire une forme presque abstraite, analytique, générant de tout autres effets. L'installation vidéo The Chittenden (2005)/19 est ainsi construite à partir d'une partition complexe, inspirée par les compositions de musique minimalistes des années 1960 et développée en collaboration avec le musicien Sean Griffin, combinant mouvements, actions et attitudes que les acteurs doivent effectuer en suivant différentes directions de jeu, effets dramatiques et postures physiques, sans pouvoir sortir des limites de ce répertoire, ni même en modifier le tempo. "Autant que construction, la théâtralité est interrogation du sens /20 ", écrivait Dort. Prisonnier de ce deus ex machina qui régit
/18 Samuel Weber (Theatricality as Medium, New York, Fordham University Press, 2004) s'intéresse à des usages non artistiques de la notion de théâtralité, notamment militaires.

/19 Cette vidéo a été présentée pour la première fois en France dans le cadre de l'exposition de la "Collection Lambert en Avignon », Figures de l'acteur, le paradoxe du comédien (8 juillet 15 octobre 2006).

/20 Bernard Dort, «La représentation émancipée », op. cit., p. 184 . 
/ $\mathbf{2 1}$ Ce retour de la figure de l'acteur sur la scène de l'art contemporain est flagrant notamment avec l'exposition «Isabelle Huppert, la femme aux portraits» (2006) ou celle déjà citée de la Collection Lambert (voir mon article " "Tirer le portrait": du visage de l'acteur et de la théâtralité (Douglas Gordon, Roni Horn, Bertrand Lavier)", Ligéia, dossiers sur l'art, XXI ${ }^{\mathrm{e}}$ année, $\mathrm{n}^{\circ}$ 81-82-8384 , janvier-juin 2008, p. 69-76.

/22 Tristan Trémeau, "Minimalisme et théâtralité, ou le "théâtre du visible". Critiques adressées par Michael Fried et Christian Bonnefoi » dans Eric Bonnet, Amos Fergombé et Edmond Nogacki (sld), Recherches valenciennoises $n^{\circ} 10$, «Théâtre et arts plastiques entre chiasmes et confluences», 2002, p. 167-177. chacun de ses gestes, l'acteur chez Sullivan se désincarne jusqu'à devenir un véritable pantin, évoquant également aux amateurs de théâtre les expériences d'une époque où le metteur en scène était tout puissant (on pense aux recherches de Craig). Vidant le réel pour ne donner à voir que l'artifice, Sullivan entend ainsi offrir une perspective sur la condition humaine à travers le prisme du théâtre et de ses masque/21, en rejoignant la déclaration d'un autre artiste de la désincarnation, Tony Oursler: "Aujourd'hui, le simulacre est aussi réel que le reste».

\section{L'objet et le spectateur théâtralisés: du «relationné », au « relationnant », au relationnel... au frontal/22?}

De tels exemples montrent, semble-t-il, une autre manifestation de la théâtralité qui s'inscrirait cette fois-ci dans une démarche réflexive de l'art visuel. Ce sont ces phénomènes qu'entend explicitement observer l'exposition «Un teatre sense teatre » conçue par Bernard Blistène et Yann Chateigné au MACBA de Barcelone, éclairant ainsi la proposition de Jessica Morgan et Catherine Wood à la Tate Modern par une approche extrêmement historicisée. En proposant un état des lieux des changements de la place du sujet (et l'émergence, notamment, d'un spectateur-sujet) à travers un dense corpus d'œuvres du $20^{\mathrm{e}}$ siècle, cette exposition, comme l'explique Manuel J. Borja-Villel, directeur du MACBA, examine méthodiquement la façon dont la théâtralité a altéré notre perception de la nature de l'œuvre d'art à travers différents dispositifs d'exposition/de (re)présentation. Le choix du titre témoigne de cette volonté de questionner cette autre scène du thêâtre sur laquelle, pour Blistène et Chateigné, quelque chose du thêâtre prend place et se libère. Car alors que le théâtre redéfinissait une «théâtralité » interne au travail scénique (pour se distinguer des autres arts et se reconstituer en tant qu'art), les observateurs extérieurs du théâtre (dont Fried fait partie) voyaient, quant à eux, non la dimension esthétique du théâtre, mais son fonctionnement global en tant que médium très spécial (la place du spectateur pour Fried, celle du public pour les lectures plus politiques), soit ce qu'une branche de l'anthropologie théâtrale a désigné comme l'exercice de la fonction dramatique - envisagé ici sans prise en compte de l'inscription sociale particulière du théâtre, ce qui ne sera pas, nous le verrons, sans conséquence.

Mais revenons une fois de plus à Fried (cité, en effet, par les quatre commissaires comme l'une des références de la conception de ces expositions). Si «Art and Objecthood» dénonçait les effets néfastes 
de la théâtralité sur l'œuvre d'art, il n'en soulevait pas moins de façon clairvoyante des questionnements sur lesquels allait se construire une partie de l'art contemporain, donnant ainsi «consistance et cohérence à la notion de théâtralité en tant que nouvelle configuration de l'expérience artistique /23 ». On a le déplacement d'une œuvre possédant des qualités de complétudes vers une construction artistique dépendante de la présence d'un regardant (ce qui amène Fried à faire presque se confondre les notions de théâtralité et d'objectité - cette condition d'objet, c'est-à-dire de non-art, de l'œuvre) et une temporalité particulière, qui n'est plus celle de l'immédiateté des œuvres modernistes, mais de la durée, interminable et infinie selon lui, de l'expérience. On sait combien les pratiques se sont développées au cours du siècle écoulé (l'exposition de Barcelone en présente un certain nombre), qui cherchent à intégrer à tout prix le spectateur afin de sortir des modes d'exposition/de présentation traditionnels. Ce phénomène, que j'ai présenté comme une volonté de dissolution du cadre (spatial et/ou temporel), se traduit sous différentes formes: d'une théâtralité «envahissante » à un minimalisme «relationnant», en passant par le démantèlement de la relation acteur/spectateur par le refus des espaces institutionnels et par l'insertion du théâtral dans la ville/vie (notamment le Living Theatre, Ben Vautier et son Théâtre de Rue, les manifestations de Daniel Buren, des membres de la Judson School dont le «NON» au théâtre n'est pas un non à la théâtralité), jusqu'à ces démarches récentes, «relationnelles». Coupées de tout contexte social, héritières d'une conception dialogique œuvre/spectateur, ces démarches sont présentées par leurs défenseurs (Nicolas Bourriaud, notamment) comme le salut permis par l'art de recréer des relations inter-humaines mises à mal par notre société marchande (les dispositifs enveloppants et dépendants d'une participation du spectateur de Dominique Gonzalez-Foerster pour ne citer qu'un exemple de la Tate). L'intérêt pour le spectateur, emprunté à la théâtralité puis passé par le prisme des arts plastiques, aboutit ainsi à des conceptions ne prenant finalement plus en compte la fonction dramatique originelle (ce qui se fera ressentir particulièrement, par ricochet, dans le champ du théâtre). L'exposition n'est plus, dès lors, interne à l'œuvre, mais « séparé[e] de son contenant (l'œuvre)», exposition du mode d'exposition donnant lieu à ce qui devient pour certains une «mise en scène de plus en plus didactique /24 ». En questionnant la frontière parfois ténue entre performeur et spectateur, le rapport regardant/regardé, ces œuvres entendent et permettent pour les meilleures de repenser de façon critique la notion de public et de communauté. Or, pour en revenir à Fried, un autre aspect déjà présent dans le texte, mais souvent oblitéré,
/23 Jacques Sato, "Littéralité et théâtralité », op. cit., p. 172.

/24 C'est la position notamment du peintre Christian Bonnefoi exposée ici par Tristan Trémeau, «Minimalisme et théâtralité, ou le "théâtre du visible" ", art. cit., p. 168. 
/25 Dans un mouvement inverse, c'est le théâtre qui cette fois-ci emprunterait, ou devrait emprunter, aux arts plastiques la fameuse dimension relationnelle.

/26 Ligéia, dossiers sur l'art, XXIe année, $n^{\circ}$ 81-82-83-84, «Art et frontalité: scène, peinture, performance ", op. cit.

concerne l'introduction d'une dialectique du regard et du visible née de la confrontation du spectateur à une œuvre "ouverte", théâtrale en ce qu'elle associe, comme le démontrera Georges Didi-Huberman, factice (objet) et présence (phénoménologie), qui nécessiterait de la part du spectateur non pas une participation physique (au sens de mouvante, actante), mais la ré-incarnation de l'œil à travers des dispositifs cette fois-ci cadrés. C'est ce qui intéressera, il me semble, un artiste comme Dan Graham qui, dans les années 1970, ne cesse de questionner l'exercice de la vision de façon radicale par le biais de dispositif-miroirs (synthèse, selon Blistène et Chateigné, entre le Pop Art et le minimalisme à travers la phénoménologie), puis par l'interrogation dès le début des années 1980 de l'espace architectural classique du théâtre qu'il confrontera à celui du cinéma. Chez Graham, le spectateur ne regarde que lui-même, mais n'est-ce pas le cas d'une certaine manière dans bien des situations? Des installations comme celles de l'artiste danois Jeppe Hein, présenté à la Tate, souvent sous la forme de cubes ou de labyrinthes réfléchissants, s'inscriront dans cette lignée, prolongeant les démarches d'artistes comme Robert Morris, Bruce Nauman, Robert Smithson ou Sol LeWitt. Mais certains dispositifs vont encore plus loin dans cette interrogation du voir en réaffirmant distinctement la séparation entre un espace du voir et un espace du jeu et en ré-individualisant le spectateur. Il est par exemple intéressant de noter dans les travaux précédemment cités de Deller ou Sullivan, qui entendent le théâtral comme une interrogation sociale et anthropologique, le maintien (ou le retour) à un dispositif frontal pensé, volontaire, laissant au spectateur sa posture de spectateur, voire accentuant la frontière regardé/regardant comme pour permettre un recul critique nécessaire, une affirmation de se trouver en face d'un espace autre. Ainsi, chez Sullivan par exemple, le retour à un dispositif archaïque du théâtre (l'un joue en face de l'autre qui regarde) dérangé par l'évidement du sens de signes traditionnellement signifiants apparaît comme une possibilité de regard critique, individuel, sur une société du spectacle, permettant justement de séparer le théâtre (l'art) de la vie par le dispositif spectaculaire. Pour finir, je dirais que de telles démarches rejoignent tout un courant actuel du théâtre qui, résistant à des propositions participatives toujours plus nombreuses (cherchant ainsi à faire du spectateur un actant $/ 25$ ), tend à réhabiliter la frontalité non dans une volonté réactionnaire mais comme une posture esthétique et politique /26.

\section{Laure Fernandez}

